\title{
Risk Factors and Strategies of Anastomotic Fistula after Sphincter-Pre- serving Surgery for Laparoscopic Rectal Cancer
}

\author{
Liang Xue \\ New Hospital of Xinyi City, Jiangsu Province, Xinyi 221400, China
}

\section{ARTICLE INFO}

Article history:

Published online: 15th July, 2017

Key words:

Laparoscopy

rectal cancer

anus preserving surgery

anastomotic fistula

\section{ABSTRACT}

Objective: To study the risk factors and strategies of sphincter-preserving surgery after laparoscopic rectal cancer for reference. Methods: 80 patients who underwent laparoscopic rectal cancer from January 1, 2015 to December 31, 2016 were the subjects of this study, and the detailed analysis of 80 patients was retrospectively analyzed. The occurrence of anastomotic fistula and the number of patients did not occur into the ratio. Results: The results showed that anastomotic fistula occurred and the body weight index did not occur, preoperative albumin and other significant differences, $p<0.05$. Conclusion: The risk factors of anastomotic fistula include body mass index, preoperative albumin and so on. It is effective to reduce the incidence of anastomotic fistula in patients with risk factors.

\section{Introduction}

The current survey data show that from 2011 onwards, the incidence of rectal cancer in China is increasing year by year, the incidence of developed areas is more prominent, the majority of rectal cancer treatment is to choose rectal cancer anus surgery, but the late operation prone to anastomotic fistula, patients need to undergo secondary surgery, which is a severe threat to the lives of patients. Therefore, this article is to explore the laparoscopic surgery for rectal cancer after anastomotic fistula risk factors and countermeasures, the research report is as follows. 


\section{Information and methods}

\subsection{General information}

Selected from January 1, 2015 to December 31, 2016 in our hospital for laparoscopic rectal cancer sphincter-preserving surgery of 80 patients for the study of the object. The patient's age between 18 to 84 years old. Male patients 63 cases and 17 cases of female patients. Weight of $56.3 \sim 81.3 \mathrm{~kg}$, the average $(56.2 \pm 2.7) \mathrm{kg}$.

\subsection{Research methods}

The data of 80 patients who underwent laparoscopic rectal cancer operation were retrospectively reviewed. The data of the patients after the operation were analyzed. The patients with anastomotic fistula were treated with the anastomotic Fistula patients were divided, and the establishment of the table to compare the two groups of data, according to the results of the comparison, the laparoscopic rectal cancer anus injury anastomotic fistula risk factors and countermeasures to discuss.

\subsection{Standards}

Patients choose to exclude criteria: surgical taboo patients, the second recurrence of patients, unconscious patients, patients with laparotomy.

Rectal cancer anus surgery anastomotic fistula diagnostic criteria: 1) Abdominal pain, abdominal distension or fever continued. 2) Gastrointestinal contrast agent outflow. 3) Abdominal incision or abdominal drainage tube out of intestinal contents. 4) Re-surgery confirmed. The above points, in line with one of the points can be diagnosed.

\subsection{Data processing}

The data of this study were statistically analyzed by SPSS 17.0 software. The relationship between anastomotic fistula and related factors in rectal cancer was count and data $(\%)$, chi-square test. $\mathrm{P}<0.05$ that the difference was statistically significant.

\section{Results}

There was a significant difference between the anastomotic fistula and the body mass index, preoperative albumin, surgical procedure, ultra-low anal retention and neoadjuvant radiotherapy and chemotherapy, $\mathrm{p}<0.05$. The difference was statistically significant. Specific data in Table 1.

\section{Conclusion}

With the continuous development of medical technology for the treatment of rectal cancer surgical methods have gradually changed. The original artificial anus is gradually replaced by anus surgery, which makes the artificial anus for the patient's psychological and physical damage is avoided, which for the recovery of patients after surgery and life has brought a great impact.

The low rectal resection surgery can reduce the appearance of anastomotic fistula, but not only will lead to a short-term life of patients will not change. It will increase the odds of secondary surgery and in recent years, the development of minimally invasive surgery more and more rapid. Laparoscopic surgery for rectal cancer, and laparotomy for patients with similar results, and because of minimally invasive surgery, the damage to patients is also smaller.

Table 1 Laparoscopic rectal cancer after sphincter-preserving surgery anastomotic fistula and the relationship between the occurrences of related factors

\begin{tabular}{|c|c|c|c|c|c|c|c|c|c|c|}
\hline \multirow[t]{2}{*}{ Item } & \multicolumn{2}{|c|}{ Body mass index } & \multicolumn{2}{|c|}{$\begin{array}{l}\text { Preoperative albu- } \\
\text { min }\end{array}$} & \multicolumn{2}{|c|}{ Surgery Method } & \multicolumn{2}{|c|}{$\begin{array}{l}\text { Ultralow sphinc- } \\
\text { ter preservation }\end{array}$} & \multicolumn{2}{|c|}{$\begin{array}{l}\text { Neoadjuvant ra- } \\
\text { diotherapy and } \\
\text { chemotherapy }\end{array}$} \\
\hline & $<25$ & $\geq 25$ & $<45$ & $\geq 45$ & ISR & Dixon & $<2$ & $>2$ & Yes & No \\
\hline Incident rate & $6.3 \%$ & $12.5 \%$ & $11.3 \%$ & $5 \%$ & $7.5 \%$ & $10 \%$ & $5 \%$ & $5 \%$ & $3.8 \%$ & $12.5 \%$ \\
\hline No incident rate & $83 \%$ & $85 \%$ & $63 \%$ & $45 \%$ & $89 \%$ & $45 \%$ & $26.3 \%$ & $41.3 \%$ & $13.8 \%$ & $80 \%$ \\
\hline $\mathrm{X}^{2}$ & \multicolumn{2}{|c|}{4.897} & \multicolumn{2}{|c|}{5.979} & \multicolumn{2}{|c|}{9.37} & \multicolumn{2}{|c|}{8.302} & \multicolumn{2}{|c|}{7.643} \\
\hline$P$ value & \multicolumn{2}{|c|}{$<0.05$} & \multicolumn{2}{|c|}{$<0.05$} & \multicolumn{2}{|c|}{$<0.05$} & \multicolumn{2}{|c|}{$<0.05$} & \multicolumn{2}{|c|}{$<0.05$} \\
\hline
\end{tabular}

Note: 80 cases of patients with anastomotic fistula in 22 patients, accounting for $27.5 \%$ of the total number. 
However, even if the harm caused by minimally invasive surgery is small, it cannot completely avoid the postoperative problems and the appearance of anastomotic fistula, so for laparoscopic rectal cancer anus surgery patients to increase prevention efforts, which including preoperative. The preparation of various conditions of the intestine with anastomotic fistula risk factors in patients with a prophylactic stoma is also a way to effectively reduce the chance of anastomotic fistula and the possibility of secondary surgery. The current foreign surgery for the anastomotic stoma and other methods of prevention is mainly on the low line, ultra-low rectal cancer patients before the cut ${ }^{[4]}$. Laparoscopic surgery for laparoscopic surgery for rectal cancer laparoscopic physicians to try to choose a higher experience to avoid sudden surgery. Less experienced physicians cannot control and more experienced laparoscopic physicians can actively participate in abdominal mirror equipment design combined with experience and the needs of surgery to design more suitable for surgery laparoscopic instruments to increase the success rate of patients with surgery.

Based on the above situation, the risk factors for laparoscopic rectal cancer sphincter-preserving surgery anastomotic fistula include body mass index, preoperative albumin, surgical approach, ultra-low anus and neoadjuvant chemotherapy, etc., after surgery. Patients to take preventive measures can effectively reduce the occurrence of the anastomotic fistula to reduce the risk of postoperative patients may be, as well as the number of secondary surgery to strengthen the treatment of patients with the effect.

\section{References}

[1] Zhang Zhaizhi, Xiao Meng Meng, Li Yan, Zhang Nengwei. Laparoscopic Rectal Cancer Anus Injury after Anastomotic Fistula Risk Factors and Countermeasures [J]. Chinese Journal of Cancer Prevention, 2016, 01 (2): 40-44.

[2] Wang Desheng, Ye Gang, Feng Ping, Ma Dekui, Qiu Changhong, Li Xiaoming, Zhang Qing. Evaluation and Prevention of Anastomotic Fistula after Laparoscopic Radical Resection of Rectal Cancer [J]. Journal of Hubei University of Science and Technology (MEDICAL Version 2016, 04 (4): 303-305.

[3] Liu Hongyan, Wang Yanhui, Zhang Ying, Zhao Hang. Rectal Cancer Double Stapler after Anus Injury Anastomotic Fistula Risk Factors [J]. Jilin Medical Journal, 2007, 11 (12): 1274-1275. 